Noise and the absolute thresholds of cone and rod vision

Donner, K.

Elsevier

1992

Vision Research. 1992. 32: 853-866

http://hdl.handle.net/1975/934

http://dx.doi.org/doi:10.1016/0042-6989(92)90028-H

Downloaded from Helda, University of Helsinki institutional repository.

This is an electronic reprint of the original article.

This reprint may differ from the original in pagination and typographic detail.

Please cite the original version. 


\title{
Noise and the Absolute Thresholds of Cone and Rod Vision
}

\author{
KRISTIAN DONNER*
}

Received 24 April 1991; in revised form 4 October 1991

\begin{abstract}
Literature data on light detection by cone and rod vision at absolute threshold are analysed in order (1) to decide whether the threshold performance of dark-adapted cone vision can, like that of rod vision, be consistently explained as limited by noise from a "dark light"; (2) to obtain comparable estimates of the dark noise and dark light of (foveal) cones and (peripheral) rods. The dark noise was estimated by a maximum-likelihood procedure from frequency-of-seeing data and compared with the dark light derived from increment-threshold functions. In both cone and rod vision, the estimated dark noise coincides with Poisson fluctuations of the estimated dark light if $17 \%$ (best estimate) of $\lambda_{\max }$-quanta incident at the cornea produce excitations. At that fraction of quanta exciting, dark lights are equivalent to 112 isomerisations per sec in each foveal cone and 0.011 isomerisations per sec in each rod. It is concluded that (1) the threshold performance of dark-adapted cone as well as rod vision can be consistently described as noise-limited, but not by postulating a multi-quantum coincidence requirement for single receptors; (2) the underlying intrinsic activity in both the cone and the rod system is light-like as regards correspondence between noise effect and background adaptation effect. One possibility is that this activity is largely composed of events identical to the single-photon response, originating in the visual pigment, in cones as well as in rods.
\end{abstract}

Retinal noise Visual sensitivity Fovea Cones Rods Sensory thresholds Purkinje shift

\section{INTRODUCTION}

In this work it is shown that the sensitivity and reliability of simple light detection by dark-adapted foveal cone vision, as well as by peripheral rod vision, can be consistently described as limited by noise from an intrinsic "dark light" (Autrum, 1943; Barlow, 1956, 1957a, 1958a), but not by postulating that a certain number of quanta must coincide in at least one of independently operating receptors for a sensation to occur (van der Velden, 1944; Bouman \& van der Velden, 1947, 1948; Brindley, 1954, 1963; Cicerone \& Nerger, 1989a,b; Vimal, Pokorny, Smith \& Shevell, 1989; Wesner, Pokorny, Shevell \& Smith, 1991). In this respect, the two receptor systems appear to be essentially similar, and comparable estimates of their detectionlimiting dark noises are derived.

The intrinsic noise that interferes with light detection is formally translated into a noise-equivalent dark light, i.e. that real background light which by its Poisson fluctuations (quantal noise) would degrade the performance of an ideal observer to the level attained by the real visual system in darkness (Barlow, 1956). A priori, this is just a way of quantifying the relevant variance and not committed to specific assumptions

*Department of Zoology, University of Helsinki, Arkadiankatu 7, SF-00100 Helsinki, Finland. about underlying mechanisms. However, a main objective of the present work is to clarify to what extent the noise source is truly "light-like". A dark light was originally postulated to account for the fact that real background lights become effective in elevating visual thresholds only from a certain intensity, as if very dim backgrounds failed to significantly add to a steady illumination intrinsic to the system (Fechner, 1860; Aguilar \& Stiles, 1954; reviewed in Barlow, 1957a). This will be referred to as the illumination-equivalent dark light. To claim validity as more than formal postulates to describe two different phenomena, the noise-equivalent and illumination-equivalent dark lights must be of equal magnitude. It is shown that in both cone and rod vision an agreement is found at photometrically realistic values for the fractions of incident photons assumed to produce excitations. Thus, the variability of light detection and the susceptibility to desensitisation by background light can be explained by the same equivalent light.

A natural (although not necessary) inference is that the dark light could be largely composed of quantal excitations in the photoreceptors identical to those caused by photons, placing the source in the visual pigment molecules (Autrum, 1943; Barlow, 1956, 1957b). For rods, this idea is basically consistent with current noise recorded in single cells in darkness (Baylor, Nunn \& Schnapf, 1984). For cones, there is no evidence 
to the contrary. However, cone noise hitherto measured in single-cell recordings (voltage noise in turtle cones: Lamb \& Simon, 1977; current noise in primate cones: Schnapf, Nunn, Meister \& Baylor, 1990) has been too strong to allow the detection reliability actually attained in foveal vision, hence may not be representative of human cone noise in vivo.

\section{ASSUMPTIONS AND ANALYSIS}

\section{Fractions of quanta incident at the cornea that produce isomerisations in cones and rods}

No useful comparison of dark light and dark noise is possible without reasonably accurate photometric estimates of the proportions of quanta entering the eye at the cornea that produce excitations in photoreceptors. This proportion, calculated at the respective wavelengths of maximum absorption $\left(\lambda_{\max }\right)$, will be denoted $Q C_{\text {rod }}$ ("rod quantum catch") for peripheral rods and $Q C_{\text {cone }}$ ("cone quantum catch") for foveal cones. Hallett (1969b, 1987), Barlow, Levick and Yoon (1971) and Barlow (1977) have previously pointed out that estimates of $Q C_{\text {rod }}$ in the region of maximum rod density must be put considerably higher than the $<0.1$ originally assumed by Hecht, Shlaer and Pirenne (1942), and Table 1 reproduces Barlow's (1977) values for the relevant factors with two alterations. First, microspectrophotometry has since suggested still higher values for the proportion of quanta absorbed by rhodopsin in rod outer segments (Bowmaker, Dartnall, Lythgoe \& Mollon, 1978; Bowmaker \& Dartnall, 1980; Dartnall, Bowmaker \& Mollon, 1983). With specific absorbances 0.015-0.023 $\mu \mathrm{m}^{-1}$ (Bowmaker et al., 1978) and outer segment length $50 \mu \mathrm{m}, 82-93 \%$ of the quanta entering rods would be absorbed rather than the $50-55 \%$ assumed by Barlow. On the other hand, the conventional upper limit 0.7 (Dartnall, 1972) for the fraction of absorbed quanta that cause excitation is preferred to the somewhat speculative 1.0 proposed by Barlow. The range of estimates for $Q C_{\text {rod }}$ then becomes $0.17-0.39$. Hallett (1987) arrived at a very similar range.

The second column of Table 1 gives corresponding values for foveal cones, based on the following considerations:

(1) The proportion of quanta entering cone outer segments is estimated on the assumption that $80 \%$ of the

TABLE 1. Photometric estimates of the proportions of $\lambda_{\max }$-quanta entering at the cornea that produce excitations in rods at $15-20^{\circ}$ eccentricity $\left(Q C_{\text {rod }}\right)$ or in a hypothetical homogeneous population of foveal cones $\left(Q C_{\text {cone }}\right)$. As Marriott's (1963) experiments used $550 \mathrm{~nm}$, which is slightly off $\lambda_{\max }$ both for L- and M-cones, the lower limit for $Q C_{\text {cone }}$ in the analysis is reduced to 0.10

\begin{tabular}{lcr}
\hline & Rods & \multicolumn{1}{c}{ Cones } \\
\hline Transmission of media & $0.50-0.75$ & $0.50-0.75$ \\
Proportion entering receptors & $0.70-0.80$ & $0.60-0.80$ \\
Absorbed by visual pigment & $0.82-0.93$ & $0.59-0.83$ \\
Fraction exciting & $0.60-0.70$ & $0.60-0.70$ \\
Total & $0.17-0.39$ & $0.11^{*}-0.35$ \\
\hline
\end{tabular}

*See heading. cross-sectional area in the central fovea is effectively occupied by cone inner segments (see Hirsch \& Curcio, 1989; Curcio, Sloan, Kalina \& Hendrickson, 1990). Axial incidence is assumed, as the cone data used (Marriott, 1963) were collected with a small (1 or $2 \mathrm{~mm}$ ) artificial pupil. The upper limit $(0.80)$ holds if all quanta are funnelled into the outer segment. The lower limit observes that the diameter of the outer segment of a foveal cone is about half the diameter of the inner segment, and the light-guiding of the inner segment may be less than perfect. Assuming that the inner segment tapers from 2.5 to $1 \mu \mathrm{m}$ over $40 \mu \mathrm{m}$ length, it is estimated that about $75 \%$ of light entering the inner segment axially will be guided into the outer segment ( $c f$. the analysis by Snyder and Pask (1973) of what proportion of light striking the inner segment wall at given angles will stay within the inner segment). The lower limit for the fraction of the light incident on the retina which passes into cone outer segments then becomes $0.75 \times 0.8=0.6$.

(2) The proportion $0.59-0.83$ absorbed by the cone pigment assumes outer segment length $35-40 \mu \mathrm{m}$ for foveal cones (Polyak, 1941) and specific absorbances between 0.011 and $0.019 \mu \mathrm{m}^{-1}$ (Bowmaker et al., 1978; Bowmaker \& Dartnall, 1980; Dartnall et al., 1983). The range overlaps with ranges estimated in psychophysical studies by Walraven and Bouman (1960) and Walraven (1966).

Cone absorbance for the stimulus wavelength used by Marriott (1963), $550 \mathrm{~nm}$, lies within $2.1 \%$ from peak in long-wavelength (L) cones and 7.6\% from peak in middle-wavelength (M) cones (Dartnall et al., 1983). The relative absorbance difference of the two cone classes is small enough at $550 \mathrm{~nm}(\mathrm{~L} / \mathrm{M}$ sensitivity ratio 1.06$)$ to justify treatment of the cones in the centre of the fovea (devoid of blue cones, see e.g. Williams, MacLeod \& Hayhoe, 1981a) as a homogeneous population. The lower-limit $Q C_{\text {cone }}$ for $550 \mathrm{~nm}$ has been taken as 0.10 (instead of 0.11 ) to correct for the slight offset from $\lambda_{\max }$.

The noise-equivalent dark light from frequency-of-seeing functions

In view of photometric estimates of quantum catch in rods and cones (Table 1), the variability of light detection in darkness is larger than can be explained by quantal fluctuations in the stimulating light. The extra variance (noise) can be formally expressed in terms of quantal fluctuations from a noise-equivalent dark light (see Introduction).

In a frequency-of-seeing (FOS) function, large variability is evident as a shallow slope. The present dark light estimation makes use of all FOS-studies known to the author where the experimental protocol has been simple enough to, supposedly, bring variability close to an "irreducible" minimum (about other sources of variability, see Discussion). These are the foveal cone data of Marriott (1963) and the peripheral rod data of Hecht et al. (1942), Baumgardt (1960), Hallett (1969a) and Sakitt (1972). The analysis gives maximumlikelihood estimates for the noise-equivalent dark light at 
any assumed value of $Q C$, using all the information contained in the data. It is assumed that the "signal" events produced by photons have to be detected against an ongoing background of identical randomly occurring "dark" photon-like events (Barlow, 1956, 1977; $c f$. Baylor, Matthews \& Yau, 1980; Baylor et al., 1984; Copenhagen, Donner \& Reuter, 1987). Dark events falling within a certain summation area and time around a flash of light are summed together with the flashinduced events, and the visual system has no means of subsequently distinguishing the two types. If the flash produces $n$ events and the number of dark events summed with these is $x$, the total number of events on which the detector makes a decision is $n+x$. The detector is assumed to apply a constant criterion $C$, such that when $n+x \geqslant C$, it decides that there has been a flash (answers "seen"). However, the numbers $n+x$ occurring on different presentations of what is nominally the same flash are subject to quantal fluctuations, characterised by a Poisson distribtuion around the mean value $N+X$. Thus, the probability that a flash which produces the mean number of excitations $N$ shall be detected is

$$
P(n+x \geqslant C)=\sum_{c}^{\infty}(N+X)^{k} \cdot e^{-(N+X)} / k !
$$

$P$ is a function of $N$ with two parameters, $C$ and $X$. The optimisation implies finding the pair of values $(C$, $X$ ) under which the complete set of recorded relative frequencies attains maximum probability. This was done by an iterative computer program which calculates the binomial probability for a particular experimental outcome given any values of $C$ and $X$ (see Copenhagen et al., 1987). A unique maximum-likelihood solution is obtained only if one of the parameters is independently known. In this case, the objective was to estimate $X$, and the mean numbers of quantal excitations assumed to result from flashes of known mean photon content was varied parametrically, covering the range of photometric $Q C$ estimates in Table 1.

Signal/noise ratio. The steepness of the FOS-function indicates the reliability of detection. A numerical measure of this is the signal/noise ratio (SNR), i.e. the mean signal required for detection scaled by the total standard deviation. Here, the signal is the mean number of stimulus-induced quantal events at the $50 \%$ detection level, $N_{t}=C-X$, to be divided by the Poisson standard deviation of stimulus-induced plus "dark" events (see Barlow, 1964; Copenhagen et al., 1987; Donner, 1989):

$$
\mathrm{SNR}=N_{t} / \sqrt{N_{t}+X}
$$

In situations where Poisson fluctuations in the numbers of stimulus-induced events constitute the dominant source of variation, this expression reduces to

$$
\mathrm{SNR}=N_{t} / \sqrt{N_{t}}=\sqrt{N_{t}}
$$

[The same equation holds for a system where a mean number of detectors $\left(N_{t}\right)$ receive equal "packages" of stimulus photons on average, and Poisson fluctuation in the number of detectors actually illuminated from trial to trial is the dominant source of variation; see Discussion.]

In the presence of a background, on the other hand, the standard deviation will include Poisson fluctuations of background quanta (mean number $B$ confusable with the stimulus) giving the expression:

$$
\mathrm{SNR}=N_{t} / \sqrt{N_{t}+X+B} .
$$

Note that: (1) the SNR is independent of the $Q C$ assumed, i.e. it can be determined by the optimisation procedure described above even when the light calibration is unknown; (2) although formally all variance is referred to fluctuations in the numbers of light-induced and "dark" quantal events, the same procedure can be used for calculating SNRs from experimental data whatever the actual sources of variability.

\section{Summation parameters}

The analysis of FOS-functions gives numbers of intrinsic photon-like events interfering with the detection of a small, briefly flashed spot of light. The underlying biological reality is a rate of events, e.g. per retinal area, photoreceptor, molecule of visual pigment, etc. Assumptions about times and areas over which noise events are summed must be made with care, since they will critically affect the relation between estimates of noiseequivalent and illumination-equivalent dark lights. Here, Riccò's area of "complete spatial summation" and Bloch's interval of "complete temporal summation" will generally be taken as the relevant ranges over which noise events are pooled with signal events. (The exception is the lower bound to spatial summation in the central fovea, since the existence of a neural Riccò area there is not undisputed, see below.) Two possible objections must be considered:

(1) It has been suggested that the discrimination of signal from surrounding noise could have better spatio-temporal resolution than indicated by the Riccò-Bloch range (Makous, 1990). The empirical basis is that stimuli which are equal in detectability may still be discriminable (Zacks, 1970), and that little summation may occur between two brief flashes which fall well within the Bloch interval (e.g. Marriott, 1963) or between two small spots which fall well within the Riccò area (Sakitt, 1971). Such findings do underline that "empirical data attest only to the similarity of the measured effect of the assorted stimuli" (Zacks, 1970). They do not, however, obviate Riccò-Bloch as the spatio-temporal range from which noise is summed with a small-brief stimulus when this range has been determined by the same task (detection of a single contiguous stimulus) as that on which the estimation of detection variability (FOS-function) is based. If detection is noiselimited and two stimuli containing the same quantity of light are equally detectable (the criterion for Riccò or Bloch limits), the same amount of noise must necessarily have been pooled with both. For example, if equalquantity stimuli of 1 and $100 \mathrm{msec}$ duration are equally reliably detected, the noise interfering with the brief stimulus must have been collected over at least $100 \mathrm{msec}$. 
(2) So-called "partial" summation in space and time (implying that larger or longer stimuli are detected at a lower intensity but greater quantity of light than small-brief ones) may, depending on the conditions of stimulation, extend far outside the Riccò and Bloch boundaries (Barlow, 1958b; Hillmann, 1958; Davila \& Geisler, 1991). It might be argued that noise from such larger areas and longer times could be "partially" pooled with a small-brief stimulus. No doubt, the description of summation by a sharply delimited circular (Riccò) area and a square-wave (Bloch) time interval is crude and the efficiency of summation in fact falls off smoothly, with true partial summation at the borders. However, the very extensive ranges of "partial" summation approximating square-root laws (see Fig. 5) are no minor border effects. On the noise-limitation hypothesis, such relations suggest that as the stimulus is enlargened (or prolonged in time) proportionally more noise is summed with it (see Barlow, 1958a). Had it been summed with the same noise as the small-brief stimulus, the threshold quantity of light would have been equal ("complete" summation would have been observed).

To summarise, when applying a model where detection is limited by linearly additive noise, there are no good reasons to assume that the spatio-temporal range over which noise is pooled with excitations from a smallbrief stimulus should differ from the Riccò-Bloch range defined by the same experimental task. The following estimates are then obtained for the extent of summation:

(1) Numbers of noise-contributing receptors. In rod vision at $15-20^{\circ}$ eccentricity, Riccò's law of complete summation has been reported to hold up to at least $1^{\circ}$ dia (one subject, Hallett, Marriott \& Rodger, 1962), $1.1^{\circ}$ (mean of 3 subjects, Weinstein \& Arnulf, 1946), $1.4^{\circ}$ (1 subject, Hallett, 1962) and $1.3,1.5$ and $1.6^{\circ}$ (3 subjects, Scholtes \& Bouman, 1977) [see also Hallett (1963) and Baumgardt (1972) for reviews]. The mean area \pm SEM is $1.3 \pm 0.2 \operatorname{deg}^{2}\left(0.11 \pm 0.02 \mathrm{~mm}^{2}\right)$. Assuming rod density $155,000 \mathrm{~mm}^{-2}\left(13,100\right.$ rods $\mathrm{deg}^{-2}$; Østerberg, 1935 ), the typical number of rods from which signals are inextricably pooled at $15-20^{\circ}$ eccentricity would be $17,000 \pm 3000$. [For Sakitt's data at $7^{\circ}$ eccentricity, this number is reduced by a factor of 0.38 due to smaller Riccò area (factor 0.5, see e.g. Hallett, 1963) and lower rod density (factor 0.75, see Østerberg, 1935).]

In the fovea, the smallest number of cones from which signal and noise events are pooled is equal to the minimum number that, due to limitations of eye optics, can be illuminated by a small-spot stimulus. This lower bound is realistic if there exists no neural Riccò area, because Piper's square-root law of spatial summation is approximated over a wide and optically reliable range of larger test spots (see below), suggesting that noise is summed over precisely the area of the stimulus (see Barlow, 1958b). Marriott (1963) assumed that the $1^{\prime}$ stimulus spot in his experiments effectively subtended $3^{\prime}$ at the retina, giving an area of $7 \mathrm{~min}^{2}$. With pupil dia 1 or $2 \mathrm{~mm}$, this roughly agrees with the foveal pointspread function of Vos, Walraven and van Meeteren (1976) and the line-spread functions of Campbell and
Gubish (1966). The psychophysical evidence and idealobserver analysis of Davila and Geisler (1991) provides a still lower estimate, $5.5 \mathrm{~min}^{2}$ (dia 2.6'), which will here be accepted as a lower bound.

The upper limit for foveal summation is also derived from Davila and Geisler (1991), who conclude that in the dark-adapted fovea there is a true neural Riccò area summing over as much as $10.4 \mathrm{~min}^{2}$. The limits for the summation area then become $5.5-10.4 \mathrm{~min}^{2}$ or, using a mean peak cone density of 199,000 cones $\mathrm{mm}^{-2}(16,850$ cones $\mathrm{deg}^{-2}$; Curcio et al., 1990), 26-49 cones, with 38 as the middle estimate. Individual variation in peak cone densities $\left(100,000-324,000 \mathrm{~mm}^{2}\right.$, Curcio et al., 1990) widens the range of uncertainty, but will be disregarded for the moment, since it affects noise-equivalent and illumination-equivalent estimates of the dark light per cone equally (see Fig. 4).

(2) Summation times. For foveal cone vision, the summation time given by Marriott (1963), $0.1 \mathrm{sec}$, is in good agreement with other estimates (Herrick, 1956; Zacks, 1970). For peripheral rod vision, Bloch's law has been reported to hold up to $0.1 \mathrm{sec}$ (Baumgardt \& Hillmann, 1961), $\geqslant 0.1 \mathrm{sec}$ (Barlow, 1958b), $0.15 \mathrm{sec}$ (Hallett, 1969a, Fig. 4) and $0.2 \mathrm{sec}$ (Sharpe, Fach \& Nordby, 1988). The range is $0.1-0.2 \mathrm{sec}$ with $0.15 \mathrm{sec}$ as the middle estimate.

Total spatio-temporal summation. The best estimate for spatio-temporal pooling of rod signals at the darkadapted threshold would be $(17,000$ rods $) \cdot(0.15 \mathrm{sec})$ $\approx 2500 \mathrm{rod} \cdot \mathrm{sec}$. The combined uncertainty in spatial and temporal summation amounts to total error limits $1100-4600 \mathrm{rod} \cdot \mathrm{sec}$ (where the spatial uncertainty is included as $95 \%$ confidence limits, the temporal uncertainty by a range). For dark-adapted foveal cone vision, the best estimate of spatio-temporal noise summation would be $(38$ cones $) \cdot(0.1 \mathrm{sec})=3.8$ cone $\cdot \mathrm{sec}$ (range $2.6-4.9 \mathrm{cone} \cdot \mathrm{sec})$.

\section{The illumination-equivalent dark light from increment- threshold functions}

The idea that an intrinsic dark background light limits absolute sensitivity in the same way as a real background light limits incremental sensitivity is formally expressed by the equation

$$
I_{\mathrm{T}}=a\left(I_{\mathrm{B}}+I_{\mathrm{D}}\right)^{b}
$$

where $I_{\mathrm{T}}$ is the intensity of the threshold increment stimulus, $I_{\mathrm{B}}$ is the intensity of the background, $I_{\mathrm{D}}$ is the intensity of the dark light, $b$ is the asymptotic slope of the threshold-vs-background function in a log-log plot, and $a$ is a scale constant. Note that this description is in no way dependent on the idea that threshold is noise-limited, but includes that as one possibility: for $b=0.5$, equation (5) describes Rose-deVries adaptation, which is the relation expected if a detector with an intrinsic background light $I_{\mathrm{D}}$ performs a signal/noise discrimination of constant reliability at all background intensities. For $b=1$, it describes Weber's law (threshold at constant contrast). It is well-known that $b$ can in fact take on any intermediate values depending on the 
conditions of stimulation. Graphically, $I_{\mathrm{D}}$ is obtained at the abscissa where the two asymptotes of equation (5) intersect in a log-log plot: the ordinate of the absolute threshold $\left(I_{\mathrm{T}}\right.$ when $\left.I_{\mathrm{B}} \ll I_{\mathrm{D}}\right)$ and the asymptote of slope $b$ describing $I_{\mathrm{T}}$ when $I_{\mathrm{B}} \gg I_{\mathrm{D}}$. This is illustrated in Fig. 1. Irrespective of the $b$-value associated with a particular stimulus configuration, the dark light $I_{\mathrm{D}}$ derived in this way will in principle be the same (for experimental support, see Barlow, 1958a; Blakemore \& Rushton, 1965).

\section{RESULTS}

Absolute thresholds and noise-equivalent dark events derived from frequency-of-seeing functions

Estimates assuming that low fractions of corneal quanta excite. Thresholds and noise-equivalent dark lights derived from FOS-functions, expressed as numbers or rates of photon-like events in receptors, depend on what $Q C$ value is assumed. Estimates of the threshold number of isomerisations from the stimulus will be proportional to $Q C$ and dark light estimates roughly to $(Q C)^{2}$. Therefore, estimations were done at several $Q C$ values, covering the ranges in Table 1 . For the sake of clarity, however, the presentation will first be based on the lower-limit values (i.e. 0.17 for rods at $15-20^{\circ}$ and 0.10 for foveal cones), giving the most conservative estimates of dark noise.

Figure 2 shows three cone functions (Marriott, 1963: left column) and three rod functions from Hecht et al. (1942), right column fitted with cumulative Poisson curves [equation (1)]. The full-drawn lines give optimal fits (corresponding to the parameters $N_{t}$ and $X$ listed in Table 2). The dotted curves correspond to $95 \%$ confidence limits for the numbers of noise events $X$, determined by keeping the threshold value $N_{t}=C-X$ fixed and finding the upper and lower $X$ values at which the probability of the experimental data has fallen to 0.05 of that under the optimal fit.

The parameters obtained from optimisations like those in Fig. 2 are given in Table 2 for the cone data of Marriott (1963, 9 subjects, fovea), together with data from 4 studies on dark-adapted rods: Hecht et al. (1942, 5 sets of data from 3 subjects, $20^{\circ}$ temporal), Baumgardt (1960, 4 subjects, $15-20^{\circ}$ eccentricity), Hallett (1969b, pooled from 3 subjects, $18^{\circ}$ nasal) and Sakitt (1972, 3 subjects on several criteria for seeing, $7^{\circ}$ temporal). The table gives the $50 \%$ response threshold $N_{t}$, the number of dark events $X$ and the dark light per receptor (see below) for each set of data. When analysing Sakitt's data, the assumed $Q C_{\text {rod }}$ has been $0.75 \times 0.17=0.13$ to correct for lower rod density at $7^{\circ}$ eccentricity (Østerberg, 1935).

The absolute threshold depends on the criterion for seeing. The mean foveal cone threshold in Table 2 is $60 \pm 4$ photoisomerisations $(n=9)$ and the mean of the rod thresholds in the fully comparable studies of Hecht et al. and Baumgardt is $16.9 \pm 1.1(n=9)$. A direct comparison of these values is not very meaningful, however. Barlow (1956) showed that, at least in rod vision, the $50 \%$ response threshold is no constant of nature, but depends on the subjectively chosen criterion for "seeing". By adopting a lax criterion ("there may have been a flash"), subjects could lower the $50 \%$ response threshold at the expense of reliability (implying a higher rate of false positives). Sakitt (1972) developed this idea more quantitatively in experiments where subjects did not simply answer "seen" or "not seen", but gave ratings $(0-6)$ for the perceived brightness of the flashes. Thus, (partly) independent sets of "seeing"

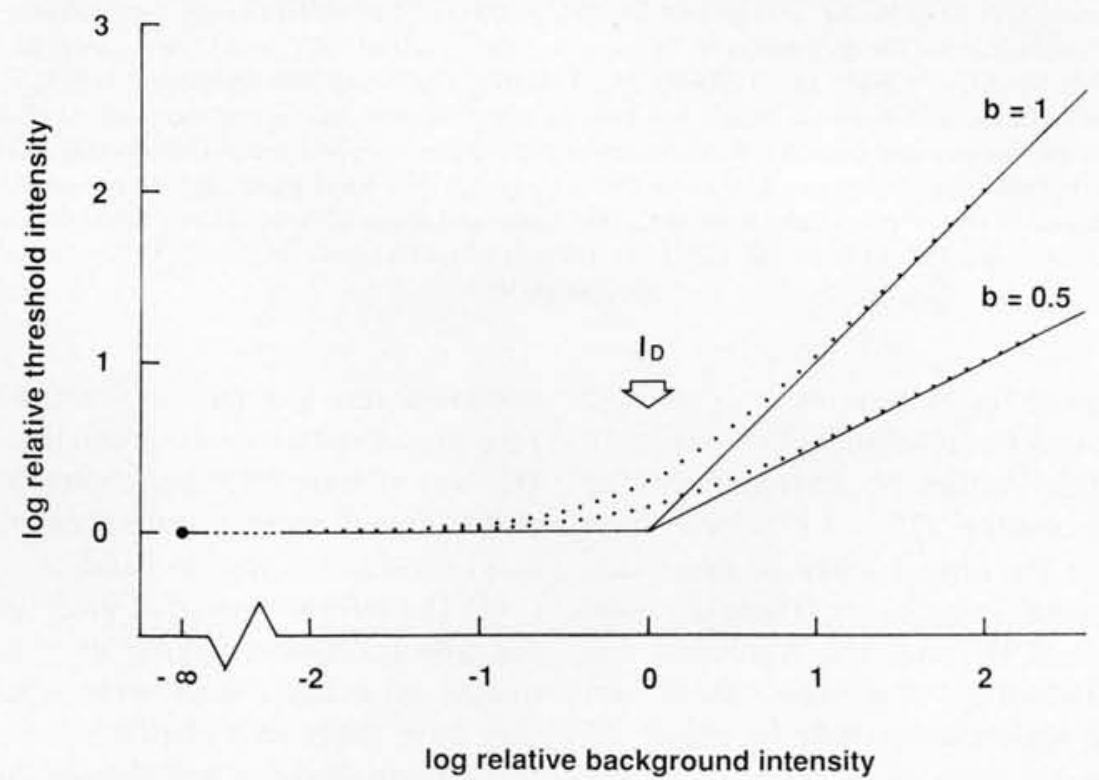

FIGURE 1. Illustration of increment-threshold functions generated by equation (5) and graphical determination of the dark-light (the constant $I_{\mathrm{D}}$ ). Shown as dotted lines are functions for $b=1$ (whereby the full-drawn asymptote describes Weber's law) and $b=0.5$ (whereby the full-drawn asymptote describes the Rose-deVries law). Abscissa, $\log I_{\mathrm{B}} / I_{\mathrm{D}} ;$ ordinate, $\log$ threshold intensity relative to the dark-adapted threshold (solid circle). Irrespective of $b$, the sloping asymptote intersects with the dark-adapted threshold level at the value $\log I_{\mathrm{D}}$ on the abscissa. 

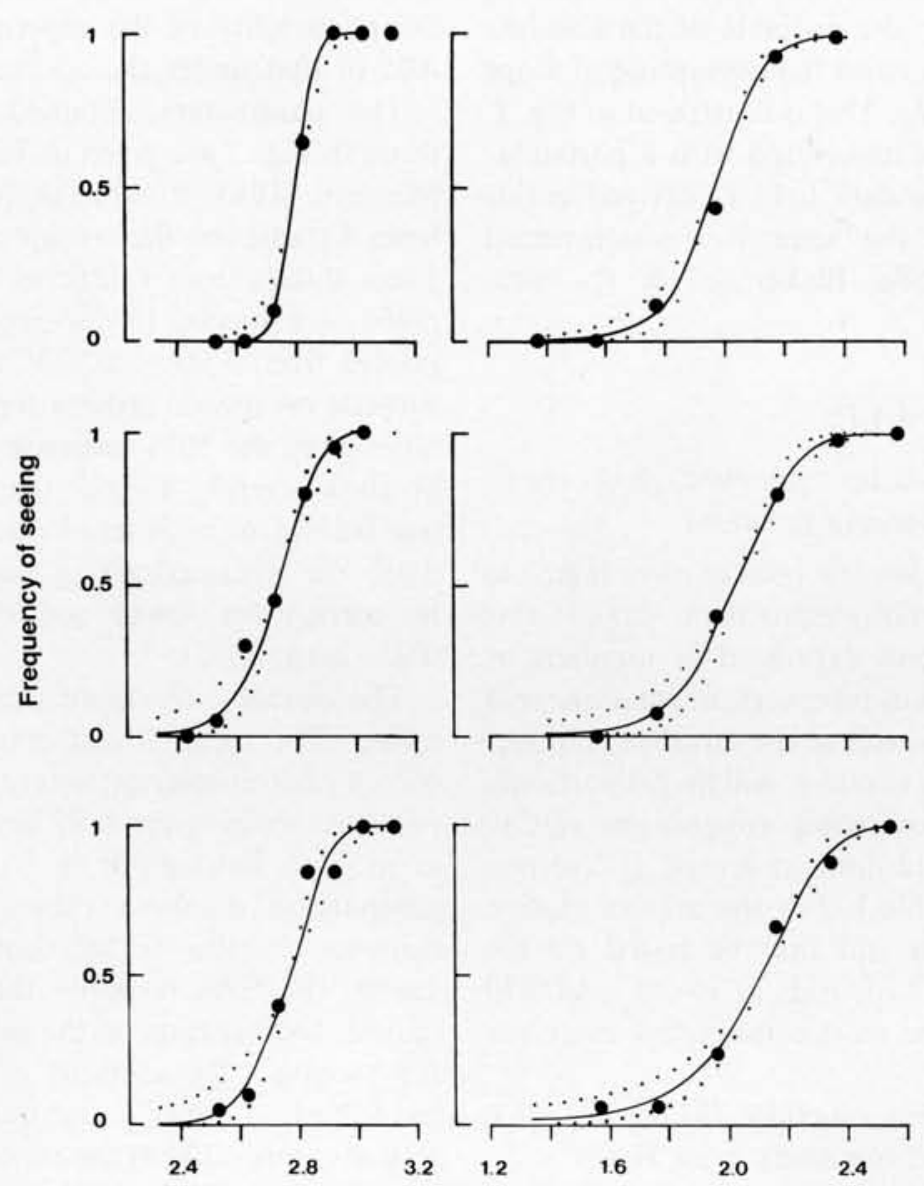

$\log$ quanta ${ }_{\lambda_{m a}}$ at cornea

FIGURE 2. Examples of dark-adapted frequency-of-seeing functions fitted with maximum-likelihood cumulative Poisson curves [equation (1)]. Left column, foveal cone vision [subjects LG, FM and DW from Marriott (1963)]; right column, rod vision at $20^{\circ}$ eccentricity [data sets SS (II), SH (II) and MHP from Hecht et al. (1942)]; abscissa, log mean flash intensity (numbers of $\lambda_{\max }$-quanta entering at the cornea); ordinate, relative frequency of flashes seen. The total number of presentations at each mean intensity was 20 in the left column and 50 (SS), 40 (SH) and 50 (MHP) in the right column. The full-drawn lines are optimal Poisson curves. The parameters $N_{t}, X$ (assuming $Q C_{\text {cone }}=0.10, Q C_{\text {rod }}=0.17$ ) are: cones: 62,0 (LG); 54,171 (FM); 57, 199 (DW); rods: 15, 7 (SS II); 18, 30 (SH II); 21, 77 (MHP). The dotted lines correspond to 95\% confidence limits, i.e. curves for which the assumed noise ( $X$-values) has been decreased (steeper curve) and increased (shallower curve) until the probabilities of the experimental data have fallen to $5 \%$ of those under the optimal fits. For observer LG (cones) the best fit had zero noise, but the upper confidence limits was 102 noise events. For SS II (rods), the steeper dotted curve has zero noise and its likelihood is $6 \%$ of that of the optimal fit. The lower and upper $95 \%$ confidence limits thus obtained for the fitting of $X$ are: cones: 0 and 102 (LG); 64 and 430 (FM); 130 and 850 (DW); rods: 0 and 22 (SS II); 10 and 70 (SH II); 43 and 140 (MHP).

frequencies were obtained for each rating: 1 or more, 2 or more etc., in rising order of reliability of detection. In Fig. 3(A), the threshold values $N_{t}$ derived from her different sets (still assuming $Q C=0.13$ ) have been plotted as functions of the ratings taken to demarcate "seeing" from "not seeing" in each case. There is a good linear correlation $\left(r^{2}=0.94\right)$, and the regression line $(y=3.49 x-0.31)$ indicates that one score on the brightness rating scale corresponds to about 3.5 photoisomerisations.

This exposition of Sakitt's (1972) data is in partial conflict with her own, since she chose to postulate that the brightness rating is equal to the number of photoexcitations received in any trial. Thereby, she had to assume a very low $Q C$ ( $c a 0.03), 4$ times lower than is here considered a realistic minimum. If it is accepted that $Q C$ was at least 0.13 , her contention that subjects are able to "count every quantum" cannot be upheld, yet her main conclusions remain valid:

(1) The information from single quanta is available at the central decision-making level: subjects can "count" quanta on a linear scale, even when not sure whether they have really seen anything.

(2) There exists no well-defined "absolute threshold"; it depends on the chosen level of reliability. The mean $50 \%$ threshold in Table 2, 16.9 isomerisations, is seen to correspond to the high brightness rating 4.9 on Sakitt's scale, presumably reflecting an ambition 
TABLE 2. Estimates of threshold numbers of flash-induced quantal events $\left(N_{1}\right)$, numbers of dark events summed with these $(X)$ and noise-equivalent dark lights (for rods, $X / 2500$ events $\mathrm{rod}^{-1} \mathrm{sec}^{-1}$, for cones, $X / 3.8$ events cone $\mathrm{e}^{-1} \mathrm{sec}^{-1}$, see Assumptions and Analysis) derived from optimal fits of equation (1) to the respective FOS-data. These estimates are based on the lowest photometrically realistic fractions of quanta producing excitations $\left(Q C_{\text {rod }}=0.17, Q C_{\text {cone }}=0.10\right)$

\begin{tabular}{|c|c|c|c|}
\hline & $N_{\mathrm{t}}$ & $x$ & $I_{\mathrm{D}}$ \\
\hline \multicolumn{4}{|l|}{ Cones } \\
\hline T.G. & 54 & 92 & 24 \\
\hline L.G. & 62 & 0 & 0 \\
\hline F.M. & 54 & 171 & 45 \\
\hline T.P.-M. & 49 & 76 & 20 \\
\hline A.S. & 70 & 88 & 23 \\
\hline J.S. & 59 & 220 & 58 \\
\hline D.T. & 48 & 65 & 17 \\
\hline J.T. & 85 & 290 & 76 \\
\hline D.W. & 57 & 199 & 52 \\
\hline Mean & $60 \pm 4$ & $133 \pm 31$ & $35 \pm 8$ \\
\hline \multicolumn{4}{|l|}{ Rods } \\
\hline \multicolumn{4}{|c|}{ Hecht et al. (1942) } \\
\hline S.H. (I) & 23 & 44 & 0.018 \\
\hline S.H. (II) & 18 & 30 & 0.012 \\
\hline S.S. (I) & 14 & 12 & 0.005 \\
\hline S.S. (II) & 15 & 7 & 0.003 \\
\hline M.H.P. & 21 & 77 & 0.031 \\
\hline \multicolumn{4}{|c|}{ Baumgardt (1960) } \\
\hline E.B. & 12 & 5 & 0.002 \\
\hline M.G. & 17 & 23 & 0.009 \\
\hline B.H. & 16 & 23 & 0.009 \\
\hline C.C. & 16 & 33 & 0.013 \\
\hline Mean & $16.9 \pm 1.1$ & $28.2 \pm 7.4$ & $0.011 \pm 0.003$ \\
\hline \multicolumn{4}{|c|}{ Sakitt (1972) } \\
\hline B.S. & Fig. 3* & 20 & 0.021 \\
\hline L.F. & Fig. $3^{*}$ & 33 & 0.035 \\
\hline K.D. & Fig. $3^{*}$ & 23 & 0.024 \\
\hline \multicolumn{4}{|c|}{ Hallett (1969) } \\
\hline 3 subjects & 16 & 15 & 0.006 \\
\hline
\end{tabular}

*Depends on the criterion for seeing.

(of experimenters and subjects) to generate steep FOScurves with a distinct threshold.

The fact that the cone FOS-curves in Fig. 2 are steeper than the rod curves indicates that the reliability criterion has been higher. Whether the cone threshold can be voluntarily lowered like the rod threshold is not known at present, but it will be argued that a comparison of rod and cone thresholds is most meaningful if it refers to a situation where both systems detect the light with the same reliability (see Discussion).

Estimates of noise-equivalent dark events are independent of the criterion for seeing. For the present purpose, however, the most important conclusion from Sakitt's data is that the estimated numbers of dark noise events is independent of the subjectively chosen threshold level.

*One of the reviewers of this paper drew my attention to the receiver-operating-characteristic (ROC) analysis of the same data by Makous (1990). There, under the assumption that $Q C_{\text {rod }}=$ 0.097 , the equivalent Poisson noise was estimated to 7-13 events (mean range for the three subjects). With the same assumed $Q C_{\text {rod }}$, the present analysis would give $12.6 \pm 1.9$ (mean \pm SEM), showing a satisfactory agreement between the two methods.

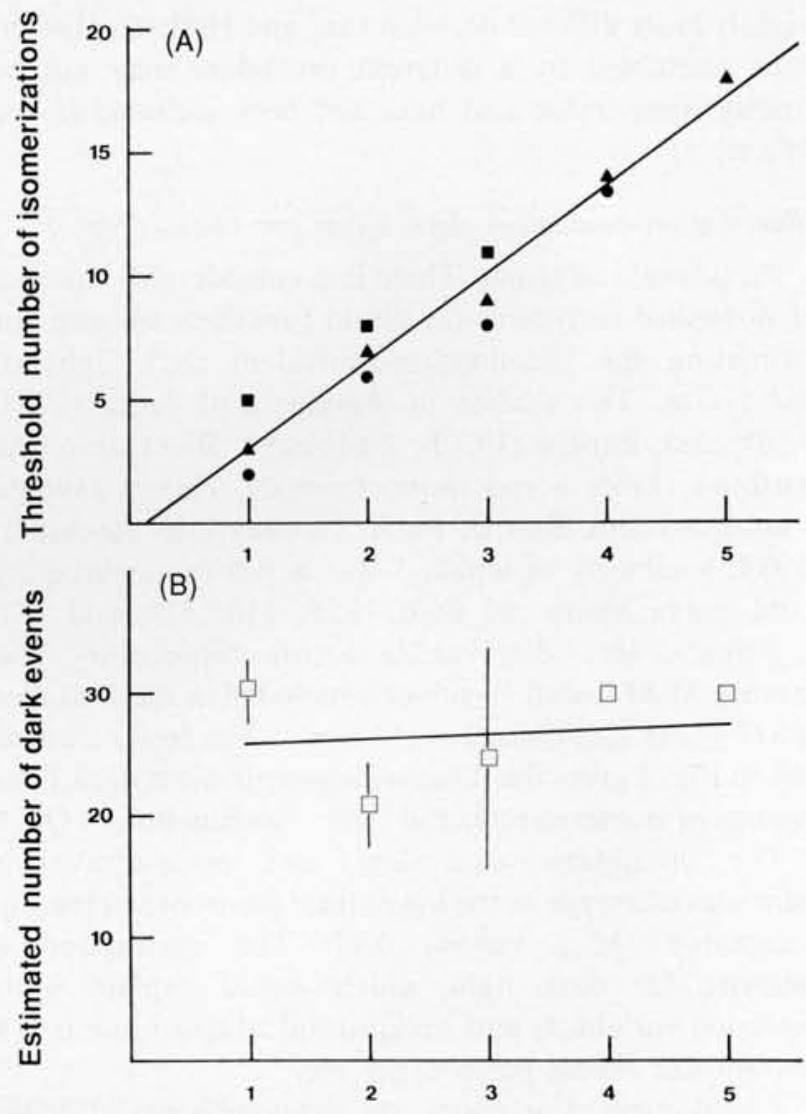

Score taken as threshold

FIGURE 3. Threshold depends on the criterion for seeing, but the estimated dark light does not. Analysis of the rod FOS-data of Sakitt (1972) assuming $Q C_{\text {rod }}=0.13$ (which corresponds to 0.17 at $20^{\circ}$ eccentricity where rod density is higher). (A) The threshold values $N_{t}$ obtained when different scores (1-5) on a subjective brightness scale were taken to demarcate seeing from non-seeing. The regression line has slope 3.5 isomerisations/brightness unit and explains $94 \%$ of the differences in $N_{t}$. (B) The noise-equivalent numbers of dark events $X$ (bars are SEMs). There is no correlation between the seeing criterion and $X\left(r^{2}=0.0037\right)$.

Figure 3(B) illustrates that there is no correlation between the criterion for seeing and the $X$ estimates from optimal Poisson fits. The data sets for all criteria appear to reflect the same intrinsic noise in a given observer. This lends credence to the hypothesis of noise-limited performance.*

\section{Noise-equivalent dark lights per photoreceptor}

By use of the summation parameters given in Assumptions and Analysis, the numbers of dark noise events $X$ were converted into dark lights per photoreceptor per sec ( $X / 2500$ for rods and $X / 3.8$ for foveal cones). For the cone data of Marriott (1963) and the rod data of Hecht et al. (1942) and Baumgardt (1960), the lower-limit estimates of Table 2 were supplemented with estimations assuming higher $Q C$ values. In Fig. 4, the mean results of fits to the cone data at $5 Q C$ values are plotted as open circles and, correspondingly, rod results at $4 Q C$ values as solid circles. The straight lines connecting the points thus give noise-equivalent dark lights of cones and rods for any photometrically realistic $Q C$ value. [Sakitt's (1972) data from a different eccentricity and based on a 
slightly more difficult decision task and Hallett's (1969b) data assembled by a different procedure may not be strictly comparable and have not been included in the figure.]

\section{Illumination-equivalent dark lights per photoreceptor}

Peripheral rod vision. There is a considerable number of published increment-threshold functions suitable for estimating the illumination-equivalent dark light of rod vision. The studies of Aguilar and Stiles (1954; 4 subjects), Barlow (1957b; 2 subjects), Blakemore and Rushton (1965; a rod monochromat), Hallett (1969b; 3 subjects) and Sharpe, Fach, Nordby and Stockman (1989; 6 subjects of which 1 was a rod monochromat) yield mean values of $800,1855,710,518$ and 670 $\lambda_{\max }$-quanta $\mathrm{sec}^{-1} \mathrm{deg}^{-2}$ at the cornea, respectively. The mean \pm SEM for all 16 subjects included in these studies is $827 \pm 146 \lambda_{\max }$-quanta $\mathrm{sec}^{-1} \mathrm{deg}^{-2}$. The lower dashed line in Fig. 4 gives the illumination-equivalent dark light in units of isomerisations $\mathrm{rod}^{-1} \mathrm{sec}^{-1}$ as function of QC.*

The illumination-equivalent and noise-equivalent estimates converge at the lower limit for photometrically acceptable $Q C_{\text {rod }}$ values, 0.17 . The corresponding estimate for dark light which would explain both detection variability and background adaptation is 0.011 photon-like events per rod per sec.

Foveal cones. For cones, the measurements of Stiles (1953) isolating the long-wavelength sensitive (L-) cone mechanism $\left(\pi_{5}\right)$ will be considered. The background intensity required to raise threshold 10 -fold corresponded to $1.15 \cdot 10^{8} \lambda_{\max }$-quanta $\mathrm{sec}^{-1} \mathrm{deg}^{-2}$ (mean value in 4 subjects, Stiles' Fig. 17; $c f$. also Barlow, 1958a). For Mcones $\left(\pi_{4}\right)$, the corresponding background intensity was similar, $\left.10^{8}\right)$. In these measurements with a large $\left(1^{\circ}\right.$ dia $)$ and comparatively long-duration stimulus $(0.2 \mathrm{sec}$, i.e. longer than the cone summation time), the asymptotic slope $b$ was typically 0.95 [see Figs 3,9 and 16 of Stiles (1953) and Fig. 1 of Sharpe et al. (1989)]. The dashed line in the top part of Fig. 4 marks the illumination-equivalent dark light of L-cones calculated from equation (5) for that $b$-value; the error bars demarcate the limits for the range 0.9-1.0. The illumination-equivalent and noise-equivalent estimates intersect at $Q C_{\text {cone }}=0.17$, well within the photometrically acceptable range (and, incidentally, the same $Q C$-value as for rods). The best estimate for the dark light becomes 112 photon-like events cone ${ }^{-1} \mathrm{sec}^{-1}$. Also shown are values derived from voltage noise recordings in single turtle cones by Lamb and Simon (1977) and current noise recordings in isolated macaque cones by Schnapf et al. (1990). Both are seen to lie above even the highest noise-equivalent estimates (see Discussion).

*The $Q C_{\text {rod }}$ scale in Fig. 4 refers to normal rod density at $15-20^{\circ}$ eccentricity. Differences in $Q C_{\text {rod }}$ which are due solely to different rod densities in the different experimental conditions (different eccentricities, achromats etc.) cause no error in the calculation of dark lights per rod from increment threshold functions. Similarly, considering only a subset of cones (e.g. L-cones) decreases $Q C_{\text {cone }}$, but distributes the lower estimated illumination-equivalent dark light on proportionally fewer cones. cones

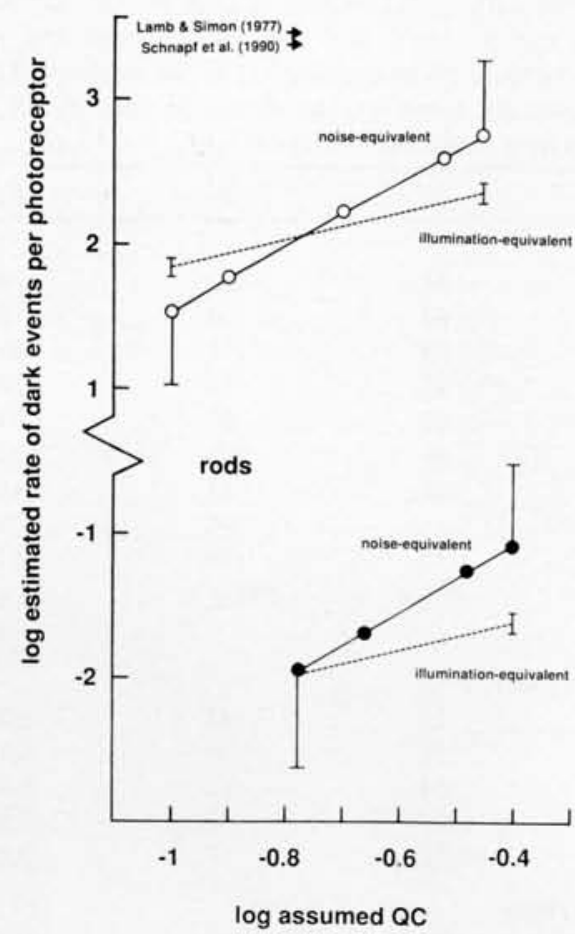

FIGURE 4. Noise-equivalent and illumination-equivalent dark lights converge at photometrically realistic $Q C$-values. Estimates of dark photon-like events per photoreceptor and sec are plotted as functions of $Q C$, the fraction of corneal quanta that produce excitations. The "data points" are the mean results of optimisations to FOS-data assuming the respective $Q C$ values, these have been connected by straight lines. Open circles refer to the cone data ( 9 subjects) of Marriott (1963), solid circles to the pooled rod data (9 subjects) of Hecht et al. (1942) and Baumgardt (1960). The dashed lines show illumination-equivalent estimates (see Text). Both for cones and for rods, the two classes of dark light estimates intersect at log $Q C \approx-0.77$ or $Q C \approx 0.17$. Also indicated (arrows) are estimates derived from dark noise in single cones by Lamb and Simon (1977) and Schnapf et al. (1990) (see Discussion). Error bars. Noise-equivalent estimates: besides $Q C$, which appears as a parameter in the figure, there are three main sources of variation: (1) individual variation in noise estimates from FOS-curves; (2) uncertainty in the spatio-temporal summation of noise; (3) individual variation in foveal cone density. The upper bound for cones (error bar at $\log Q C=-0.46$ ) was obtained by using the upper-limit estimate for factor (1) and the lower-limit estimates for (2) and (3); correspondingly, the lower bound for cones (error bar at $\log Q C=-1.0$ ) by using the lower-limit estimate for factor (1) and upper-limit estimates for (2) and (3). In these calculations, the limits used for factors (1) and (3) were the $95 \%$ confidence limits and for factor (2) the range.

The error limits for the noise-equivalent estimates for rods were calculated as for cones, except that individual variation in rod density was neglected.

Illumination-equivalent estimates: the error bar for cones shows the limits for $0.9<b<1.0$, that for rods the SEM of the literature values (see text).

Cone threshold performance cannot be explained by multiquantum coincidence in independent receptors

Detection in cone vision is still frequently analysed on the alternative "coincidence" threshold hypothesis assuming that a criterion number of photons must coincide in at least one of independently operating cones for a visual sensation to occur (see Introduction). It seems necessary to state here in detail the grounds 
for rejecting this model, because it would render the present estimation of dark noise from FOS-functions meaningless. The main argument is that orders of coincidence required to explain the area-threshold relation on one hand and the variability of light detection on the other hand are incompatible. This contradiction is summarised in Fig. 5.

The reliability of foveal light detection would require an order of coincidence no less than 6. In panel (A), the average experimental FOS-function of Marriott's 9

(A)

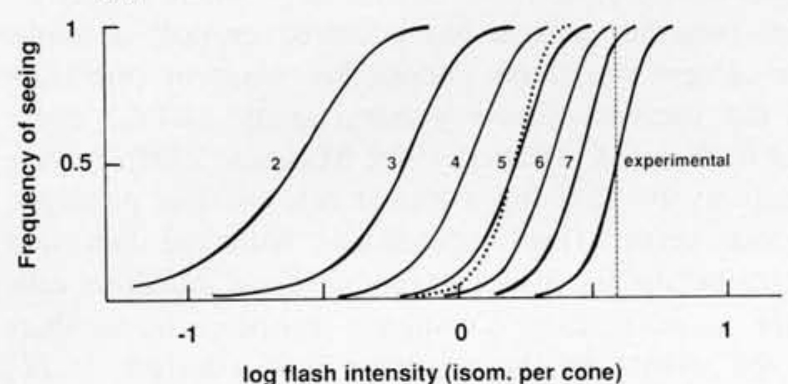

(B)

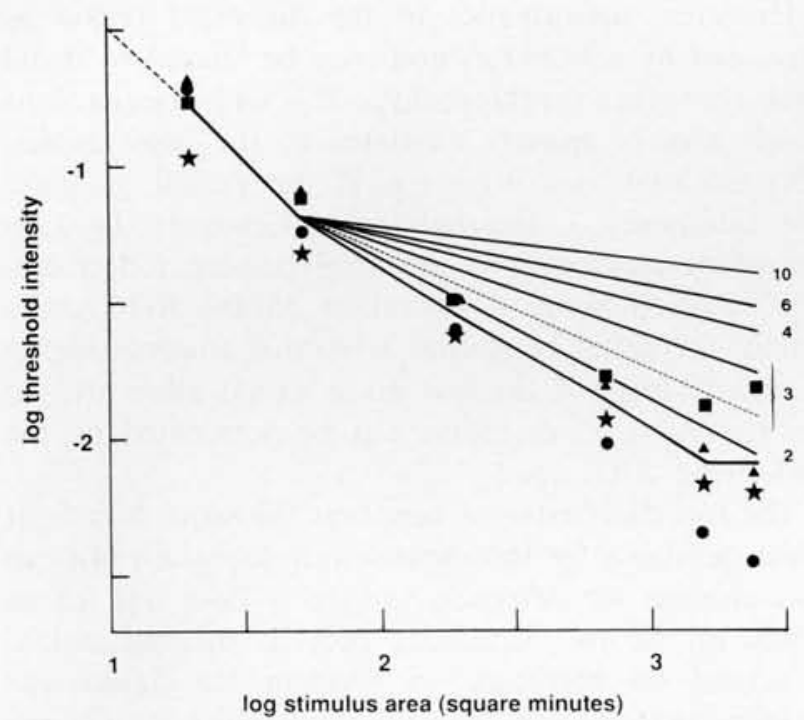

FIGURE 5. Contradiction between the orders of quantal coincidence in single cones that would be required to explain (A) FOS-functions and (B) area-threshold functions in the fovea. (A) The rightmost curve is the average experimental FOS-curve from Marriott's (1963) 9 subjects $\left(N_{t}=102\right.$, indicated by vertical dashed line, $\left.X=424\right)$. The numbered curves are the steepest that can be obtained if detection is based on 26 independent cones subject to the coincidence requirements indicated by the numbers $(2,3,4,5,6$ and 7$)$. The dotted curve is Brindley's (1963) limiting curve for 5-fold coincidence $P=1-\exp \left(-N I^{5} / 5\right.$ !) (see text). (B) How the foveal flash threshold depends on the area of the stimulating spot. Abscissa, log stimulus area (square minutes); ordinate, log threshold intensity (millilamberts for the experimental points, relative units for the calculated lines). Data points from Hillmann (1958) (10 msec flash duration, white light, natural pupil). The lowest line connects the mean thresholds for the 4 observers; this area-threshold relation follows the slope 0.6 from $8^{\prime}$ to $45^{\prime}$ spot dia. The numbered lines show the slopes predicted if threshold implies that at least one of the cones covered by the stimulus receives the number of photons indicated. The dashed line shows how the slope for 3-fold coincidence would be affected by increased cone quantum catch (see text). For easier comparison of slopes, the calculated functions have all been vertically positioned to coincide at the end of the "Riccò" range of the experimental relation (see text). subjects (for $N_{t}=102$ and $X=424$, the mean optimal parameters for $Q C=0.17$ ) is shown together with the FOS-curves that would result if 26 illuminated cones (see Assumptions and Analysis) acted as independent detectors subject to the threshold condition that 2, 3, 4, 5,6 or 7 photons coincide in at least one cone. The curves for less than 6-fold coincidence are significantly shallower than the experimental average, hence 6 is the lowest order of coincidence compatible with the shape of the FOS-function. [Shown for comparison (dotted) is the steepest curve (assuming "many" detectors) that can be obtained with a 5-fold coincidence requirement according to Brindley's (1963) formula, which has often been used as a yardstick. It resembles the experimental average curve and the curve for 7 -fold coincidence in 26 cones.] A similar conclusion is independently reached by considering the sensitivity of light detection. The mean $50 \%$ threshold among Marriott's subjects was 606 $\lambda_{\max }$-quanta entering at the cornea. If threshold were synonymous with at least 4-fold coincidence in at least one of 26 cones, this would mean that $Q C=0.05,5$-fold coincidence $Q C=0.07,6$-fold coincidence $Q C=0.10$, and 7-fold coincidence $Q C=0.12$. In view of the photometrical limits for $Q C_{\text {cone }}$ (Table 1), the lowest order of coincidence that could explain the insensitivity of dark-adapted cone vision is 6 .

The foveal area-threshold relation is incompatible with orders of coincidence higher than 2. Figure 5(B) plots area-sensitivity data for foveal $10 \mathrm{msec}$ flash stimuli presented to dark-adapted observers (lowest line; Hillmann, 1958) together with predictions from the coincidence hypothesis (lines marked by numbers). The apparent Riccò range extending to log area $1.7\left(50 \mathrm{~min}^{2}\right.$ or $8^{\prime}$ dia) may here be disregarded, as optical factors are likely to have played a significant role. The important observation is that from there up to log area $=3.2\left(45^{\prime}\right.$ spot dia), log threshold intensity falls along a mean slope of -0.6 , close to the square-root (Piper) law which would result from noise-limited detection by an ideal observer. This has been confirmed in a recent study by Davila and Geisler (1991) both for the dark- and the light-adapted fovea. For stimuli between $5.5 \mathrm{~min}^{2}$ (the optical resolution limit) and at least $256 \mathrm{~min}^{2}$, the mean (log threshold)-(log stimulus area) regression slope for their light-adapted subjects was -0.55 .

The lines marked by numbers in Fig. 5(B) show predicted area-threshold relations for "coincidence" thresholds. From the shallowest to the steepest line, it has been assumed that $10,6,4,3$ and 2 photons must coincide in at least one cone for a visual sensation to occur. Only the line for 2 -fold coincidence is at all consistent with the experimental relation.

Admittedly, the lines have been calculated on the assumption of constant cone inner segment cross section (i.e. quantum catch) over the retinal region stimulated. In reality, cone centre-to-centre spacing increases 1.5 fold from the centre of the fovea to $26^{\prime}$ eccentricity (Hirsch \& Curcio, 1989). However, even under the extreme assumption that the quantum catch of all the illuminated cones would grow by the full factor 
$(1.5)^{2}=2.25$ as stimulus area is increased, the areathreshold relation for, e.g. 3-fold coincidence would reach a slope of no more than -0.43 [dashed line in Fig. 5(B)], far from enough to reconcile it with the experimental slope.

To summarise, FOS-functions require orders of coincidence of 6 or more, but spatial summation is compatible with no more than 2. Thus, when light is delivered through the natural refractory apparatus of the eye, foveal thresholds are not determined by independently operating cones requiring a multiple hit in at least one. This is true in both dark- and light-adapted states.

Shapes of FOS-curves give no consistent information on orders of coincidence. The inadequacy of the coincidence hypothesis is further evident from foveal FOS-functions recorded under background illumination:

(1) Functions recorded under progressively stronger backgrounds (elevating thresholds by $0.2,0.5$ and $0.8 \mathrm{log}$ units from the dark-adapted value) do not differ systematically in steepness (Fig. 3 in Cicerone \& Nerger, 1989a). Yet, the coincidence hypothesis (in simple form) would require that the very different photon statistics be evident as differences in curve shapes.

(2) In the presence of background lights, detection necessarily implies that flashes are discriminated from background photon noise. The univariance principle means that a single cone cannot know which photons arriving during the $50 \mathrm{msec}$ stimulus pulse come from the stimulus and which from the background. Cicerone and Nerger (1989a), for example, usually employed background intensities $\left(I_{\mathrm{B}}\right)$ which raised thresholds by $0.5 \mathrm{log}$ units. Equation (5) with $b=0.5$ (small-brief stimulus, see Barlow, 1958a) and $I_{\mathrm{D}} \geqslant 70$ events cone ${ }^{-1} \mathrm{sec}^{-1}$ [Stiles' (1953) value if $Q C=0.1]$ shows that $I_{\mathrm{B}}$ must have been $\geqslant 620$ events cone $\mathrm{e}^{-1} \mathrm{sec}^{-1}$. Thus, an average of at least 31 background events, with a SD of 5.6 events, would have occurred in each cone within the time interval of the flash. Assuming a threshold criterion of 6 flash-induced events in at least one cone (as used by the authors for the analysis), a cone would produce a visual sensation when counting a total of $31+6=37$ events. In every cone, this would happen on every seventh $50 \mathrm{msec}$ period $[P(x \geqslant 37 \mid X=31)=0.14]$, causing an unacceptable rate of false responses.

\section{DISCUSSION}

Noise-equivalent and illumination-equivalent dark lights agree

For both cone and rod vision, noise-equivalent and illumination-equivalent dark lights were found to converge at photometrically realistic values for the fraction of corneal photons that produce excitations $(Q C)$. This suggests, firstly that both reflect a common physiological reality, secondly, that the corresponding noise sets a level of irreducible intrinsic variability (see below) in light detection by the dark-adapted cone and rod systems. The best agreement for both rods and foveal cones was reached at $Q C \approx 0.17$, whereby the equivalent dark lights would be 0.011 dark events per rod per sec and 112 dark events per cone per sec. The main conclusion - that an agreement is foundis robust, but the uncertainty of the numerical estimates is considerable, as indicated by the ranges in Fig. 4.

\section{In what sense is threshold performance noise-limited?}

The threshold performance of rod vision detecting small brief flashes is noise-limited in a strong sense, as is evident from Sakitt's (1972) experiments. They show that the full information on light-induced and dark events is accessible at the central stage where the judgement (whether a flash has occurred or not) is made. The subject can freely choose her point of operation on the receiver-operating-characteristic (ROC) curve (Cohn, Green \& Tanner, 1975; Makous, 1990), buying sensitivity by allowing a higher rate of false positives, or vice versa. This is consistent with the fact that the spike discharges of some sensitive ganglion cells in the cat retina carry a complete record of the numbers of rod events in the receptive field (Barlow et al., 1971).

However, performance in the threshold region as expressed by a FOS-function may be limited by distal noise even when the threshold itself is set by mechanisms which may be entirely unrelated to the noise aspect. This situation can arise e.g. if the retinal ganglion cell interposes a thresholding nonlinearity between the photoreceptors and the level passing judgement. The subject's point of operation on the ROC curve cannot thereafter be shifted arbitrarily towards higher sensitivity (even if the low noise would allow it), yet the variability in detection can be dominated by the underlying distal noise.

The fact demonstrated here that the same dark light which accounts for threshold-setting [equation (4)] can also account for detection variability does not tell us which of the two situations prevails in cone vision. It would be necessary to perform the Sakitt-type experiment to see if the sensitivity/reliability balance can be voluntarily redressed, or whether an independent distal thresholding mechanism intervenes. Both Marriott (1963) and Wesner et al. (1991) hint that it was possible to generate steeper curves by adopting more stringent threshold criteria, suggesting that the situation is not unlike that in rod vision.

\section{The threshold difference of cone and rod vision}

Intrinsic noise is of course generally detrimental to light detection, but its effect in an experimental situation much depends on what kind of stimulus is to be detected. The foveal cone system has the potential for very restricted spatial summation (e.g. MacLeod, Williams \& Makous, 1985). Therefore, its strong dark light does not in principle prevent it from detecting small numbers of quanta.

In the experimental data considered, the mean absolute thresholds in terms of $\lambda_{\max }$-quanta entering at the cornea were 99 for rods and 606 for foveal cones, 
estimated to correspond to 17 and 102 isomerisations, respectively. As explained above, however, any fair comparison of rod and cone thresholds should refer to detection with equal reliability, i.e. equal signal/noise ratio [SNR, equation 2]. The average SNR at the rod threshold was $17 / \sqrt{ }(17+28)=2.5$ and that at the cone threshold $102 / \sqrt{ }(102+424)=4.4$. To reach $\mathrm{SNR}=4.4$, the rod system would need 35 isomerisations, implying that sensitivies would differ by no more than a factor of $102 / 35=2.9$ ( $c f$. Arden \& Weale, 1954). Further, if the foveal stimulus could be more sharply focused, noise from fewer cones might be summed with the stimulus, and threshold might be even lower. The expected threshold of a single cone detecting a brief stimulus with the same reliability as rods $(\mathrm{SNR}=2.5)$ would be 12 isomerisations ( $70 \lambda_{\max }$-quanta at the cornea). Interestingly, this is roughly the number of isomerisations thought to suffice for detection mediated by a single blue cone (Williams, MacLeod \& Hayhoe, 1981b).

\section{The physiology of dark noise in rods and cones}

Dark photon-like events in rods. The conclusion that the dark light resembles real light in its correspondence between noise effect and adaptation effect invites speculation on its physiological nature. It is possible to identify the dark light of the rod system with the spontaneously occurring discrete photon-like events observed in the photocurrent of single rods (Baylor et al., 1980, 1984). A rod dark light of 0.011 events $\mathrm{rod}^{-1} \mathrm{sec}^{-1}$ would be well explained by the photon-like events recorded by Baylor et al. (1984) in single rods of Macaca fascicularis (rate $0.0063 \mathrm{sec}^{-1}$ in a rod whose outer segment volume is about half that of a human rod). This would place the source of the dark light in the visual pigment molecule itself (Autrum, 1943; Barlow, 1956).

The origin of cone noise. Dark noise in single cones has been measured by Lamb and Simon (1977; membrane voltage in turtle cones) and Schnapf et al. (1990; dark current in macaque cones). The former authors estimate the noise power in the frequency band that would necessarily interfere with light detection to correspond to an equivalent rate of 2800 isomerisations cone ${ }^{-1} \mathrm{sec}^{-1}$ (room temp), but conclude from the shape of the power spectrum that it is not composed of photon-like events. Schnapf et al. (1990) allow that the noise in the relevant frequency band could be composed of photon-like events and estimate the equivalent rate to 2400 events cone $^{-1} \sec ^{-1}$ (at $37^{\circ} \mathrm{C}$ ). Both estimates are more than 20 times larger than the present best estimate for human foveal cones and indeed exceed even the absolute upper bound derived here (see Fig. 4: the upper limit for the noise-equivalent dark light at the extreme $Q C_{\text {cone }}=0.35$ is ca 1900 events cone $\left.\mathrm{sec}^{-1}\right)$. Thus, the noise measured by Lamb and Simon (1977) or Schnapf et al. (1990) would preclude the reliability of light detection actually attained and is not representative of human foveal cones in vivo. Note that this discrepancy is only exacerbated under the coincidence hypothesis: for detection with the average reliability of Marriott's subjects $(\mathrm{SNR}=4.4)$, the threshold order of coincidence of an independently operating macaque cone with the noise recorded by Schnapf et al. (1990) would, according to equation (2), have to be 78 (not 6).

At present, there is no evidence against the hypothesis that the detection-limiting noise in the dark-adapted cone system could also largely consist of photon-like events initiated in the visual pigment molecule.

Is the dark event rate related to the spectral sensitivity of the visual pigment? Under that assumption, Barlow (1957b) proposed that shifting the spectral sensitivity of a visual pigment towards shorter wavelengths would decrease the dark noise by increasing the thermal stability of the pigment. The signal/noise advantage thus conferred upon rods as compared with long- and middlewavelength ( $\mathrm{L}$ - and $\mathrm{M}$-) cones would explain the otherwise paradoxical Purkinje shift. The general idea, due to Stiles (1948), is that longer-wavelength sensitivity implies a lower energy barrier for excitation of the pigment molecule, hence a higher probability of thermal excitation. A simple quantitative relation can be derived from classical statistical mechanics [equation (2) in Barlow, 1957b]. How do the present estimates fit with that theory? Remarkably, the best estimate of the L-cone/rod ratio of dark events per pigment molecule turns out to agree within a few percent with the predicted ratio $[44,000$; the calculation was based on the distribution of $\lambda_{\max }$-values in individual L-cones shown in Fig. 1 of Dartnall et al. (1983) and pigment densities given in the same work]. It is unclear what significance should be attached to this agreement. Direct measurements of the dark current of rods with different absorption maxima have indicated that despite a general correlation between red-sensitivity and a high rate of dark events, the quantitative formulation of Stiles and Barlow is not generally valid (see Firsov \& Govardovskii, 1990; Donner, Firsov \& Govardovskii, 1990). The empiristic conclusion at this stage is that dark noise tends to correlate with long-wavelength sensitivity, and this may be the "reason" for the Purkinje shift, but the physical mechanism is not simple.

\section{Rejection of the multi-quantum coincidence hypothesis}

Rejection of the coincidence hypothesis entails no claim that the neural pathway from a single cone is inherently incapable of setting its own threshold. In the primate fovea each cone is, on average, served by three ganglion cells of which two are thought to connect to that cone exclusively; if it were possible to illuminate a single cone, it would undoubtedly act as an independent detector requiring a certain order of photon coincidence (Wässle, Grünert, Röhrenbeck \& Boycott, 1989; Kolb, 1991; MacLeod et al., 1985; cf. also Williams et al., 1981b). It is quite clear, however, that foveal vision has an extensive capacity for pooling cone signals (Hillmann, 1958; Davila \& Geisler, 1991; see Fig. 5) and since eye optics will spread the image of even a point source over many cones, this spatial summation will in reality always be significant for simple detection.

Neither is it claimed that there is not, in fact, multiquantum coincidence in single cones at the absolute 
threshold: if threshold corresponds to 102 isomerisations distributed on 26 cones, then on average 23 cones will receive at least 2 isomerisations, and 5 cones will receive at least 6 isomerisations.

What is rejected is the idea that light detection in cone vision is subject to the necessary condition that a certain number of quanta coincide in at least one cone. Consequently, analytical procedures based on that assumption must be regarded with scepsis, even if the logic of the experiments is sound.

Other sources of variability than the dark light affecting FOS-functions

A central argument in this work is that the variability of light detection in some "stripped" experimental conditions approaches an irreducible level which is due only to light-like neural noise plus quantal fluctuations in the stimulus. Still, it should be realised that a dark-light estimation from FOS-functions in principle always gives an upper bound, since the functions accumulate all variability in the experimental situation. Thus, Marriott's (1963) is the only foveal study known to the author where the protocol was simple enough to believably minimise other variability, and it is instructive to consider why this should be so, i.e. how sensitivelyand at what points - extra variability may enter in other studies:

(1) Cicerone and Nerger (1989a) observed that Mcone FOS-functions are shallower than corresponding L-cone functions for detection of small-brief ( $1^{\prime}$, $50 \mathrm{msec}$ ) stimuli. In the present conceptual framework, this may be expressed as a signal/noise difference [equation (4)]: their mean SNR was 2.4 for detection by M-cones (range 2.0-2.8 in 6 subjects) and 3.2 for L-cones (range 2.9-3.7). The authors' quantitative derivation of the L:M-cone ratio $\approx 2: 1$ was largely built on the (fallacious) coincidence hypothesis. However, an alternative interpretation which preserves the main logic of the work is that their FOS-functions recorded under $\mathrm{L}$ - and M-cone isolation conditions express a variability which is predominantly due to random variation in the (small) numbers of cones of either type covered by the stimulus from flash to flash. Assume, for example, that the $\mathrm{L}: \mathrm{M}$ ratio is indeed $2: 1$, that a total of 26 cones are illuminated (i.e. $9 \mathrm{M}$-cones and $17 \mathrm{~L}$-cones) and that the two cone types are randomly shuffled. Then equation (3) shows that, due to this source of variability alone, M-cone SNR would be limited to $\leqslant \sqrt{ } 9=3$, while L-cone SNR could reach $\sqrt{ } 17=4.1$. Slopes of FOSfunctions could really carry information on relative cone densities (see, however, below).

(2) Vimal et al. (1989) and Wesner et al. (1991) show results on the detection of two small spots presented at varying locations in the dark-adapted fovea. Simple probability algebra allows extraction of detection functions for a single spot from their composite seeing frequencies. They indicate SNRs of 3.0 (RP), 2.4 (RV) and $1.3(\mathrm{YY})$ for the detection of a small $546 \mathrm{~nm}$ spot, to be compared with the average SNR of Marriott's detection functions, 4.4 . It is unlikely that their $50 \%$ threshold (signal) was that much lower than Marriott's, since they tried to avoid false positives (Wesner et al., 1991). Rather, the variability ("noise") must have been larger, conceivably reflecting an uncertainty component associated with the more complicated decision process in the two-spot experiment.

(3) Hallett (1969a, 1987) has convincingly shown that (rod) FOS-functions from long sessions contain extra variability due to drift in the threshold criterion and has devised a procedure for eliminating this component. The main reason for preferring here the long sessions of Hecht et al. (1942) and Baumgardt (1960) is that they are directly comparable to the cone data of Marriott (1963). If, however, Hallett's (1969a) corrected estimate is regarded as a purer measure of the dark light, the best convergence of rod noise- and illumination-equivalent dark lights is reached at $Q C_{\text {rod }}=0.26$, giving 0.016 events $\operatorname{rod}^{-1} \mathrm{sec}^{-1}$.

Considering how easily extra variability is incurred it is truly amazing that the area-threshold relation in the fovea should approximate that of an ideal detector (Davila \& Geisler, 1991). This implies that the detection mechanism is able to "zoom in" exactly on the stimulated region, maximising the SNR. It is hard to believe that this could be possible without a prior inner model of the stimulus to be detected, and it would be interesting to study spatial summation with randomised order of presentation of different-sized stimuli.

Finally note that for many of the works considered above (Cicerone \& Nerger, 1989a,b; Vimal et al., 1989; Wesner et al., 1991), information on absolute light intensities is not available. It must therefore be emphasised that if the detection criterion in cone vision, as in rod vision, can be voluntarily reset ( $c f$. Fig. 3), the shape of a FOS-function is not, without information on absolute light intensities, a stable measure of intrinsic variability.

\section{REFERENCES}

Aguilar, M. \& Stiles, W. S. (1954). Saturation of the rod mechanism of the retina at high levels of stimulation. Optica Acta, I, 59-65.

Arden, G. B. \& Weale, R. A. (1954). Nervous mechanisms and dark-adaptation. Journal of Physiology, 125, 417-426.

Autrum, H. (1943). Über kleinste Reize bei Sinnesorganen. Biologisches Zentralblatt, 63, 209-236.

Barlow, H. B. (1956). Retinal noise and absolute threshold. Journal of the Optical Society of America, 46, 634-639.

Barlow, H. B. (1957a). Increment thresholds at low intensities considered as signal/noise discriminations. Journal of Physiology, 136, 469-488.

Barlow, H. B. (1957b). Purkinje shift and retinal noise. Nature, 179, 255-256.

Barlow, H. B. (1958a). Intrinsic noise of cones. In Visual problems of colour (Vol. II, pp. 617-630). London: Her Majesty's Stationery Office.

Barlow, H. B. (1958b). Temporal and spatial summation in human vision at different background intensities. Journal of Physiology, 141, 337-350.

Barlow, H. B. (1964). The physical limits of visual discrimination. In Giese, A. C. (Ed.), Photophysiology (Vol. 2, pp. 163-202). New York: Academic Press. 
Barlow, H. B. (1977). Retinal and central factors in human vision limited by noise. In Barlow, H. B. \& Fatt, P. (Eds), Vertebrate photoreception (pp. 337-358). London: Academic Press.

Barlow, H. B., Levick, W. R. \& Yoon, M. (1971). Responses to single quanta of light in retinal ganglion cells of the cat. Vision Research 3 (Suppl.), 87-101.

Baumgardt, E. (1960). Mesure pyrométrique du seuil visuel absolu. Optica Acta, 7, 305-316.

Baumgardt, E. (1972). Threshold quantal problems. In Jameson, D. \& Hurvich, L. M. (Eds), Handbook of sensory physiology (Vol. VII/4, pp. 29-55). Berlin: Springer.

Baumgardt, E. \& Hillmann, B. (1961). Duration and size as determinants of peripheral retinal response. Journal of the Optical Society of America, 51, 340-344.

Baylor, D. A., Matthews, G. \& Yau, K.-W. (1980). Two components of electrical dark noise in toad retinal rod outer segments. Journal of Physiology, 309, 591-621.

Baylor, D. A., Nunn, B. J. \& Schnapf, J. L. (1984). The photocurrent, noise and spectral sensitivity of rods in the monkey Macaca fascicularis. Journal of Physiology, 357, 575-607.

Baylor, D. A., Nunn, B. J. \& Schnapf, J. L. (1987). Spectral sensitivity of cones of the monkey Macaca fascicularis. Journal of Physiology, $390,145-160$.

Blakemore, C. B. \& Rushton, W. A. H. (1965). Dark adaptation and increment threshold in a rod monochromat. Journal of Physiology, $181,612-628$.

Bouman, M. A. \& van der Velden, H. A. (1947). The two-quanta explanation of the dependence of the threshold values and visual acuity on the visual angle and the time of observation. Journal of the Optical Society of America, 37, 908-919.

Bouman, M. A. \& van der Velden, H. A. (1948). The two-quanta hypothesis as a general explanation for the behaviour of threshold values and visual acuity for the several receptors of the human eye. Journal of the Optical Society of America, 38, 570-581.

Bowmaker, J. K. \& Dartnall, H. J. A. (1980). Visual pigments of rods and cones in a human retina. Journal of Physiology, 298, 501-511.

Bowmaker, J. K., Dartnall, H. J. A., Lythgoe, J. N. \& Mollon, J. D. (1978). The visual pigments of rods and cones in the rhesus monkey, Macaca mulatta. Journal of Physiology, 274, 329-348.

Brindley, G. S. (1954). The order of coincidence required for visual threshold. Proceedings of the Physiological Society, 67B, 673-676.

Brindley, G. S. (1963). The relation of frequency of detection to intensity of stimulus for a system of many independent detectors each of which is stimulated by a $m$-quantum coincidence. Journal of Physiology, 169, 412-415.

Campbell, F. W. \& Gubisch, R. W. (1966). Optical quality of the human eye. Journal of Physiology, 186, 558-578.

Cicerone, C. M. \& Nerger, J. L. (1989a). The relative numbers of long-wavelength-sensitive to middle-wavelength-sensitive cones in the human fovea centralis. Vision Research, 29, 115-128.

Cicerone, C. M. \& Nerger, J. L. (1989b). The density of cones in the fovea centralis of the human dichromat. Vision Research, 29, $1587-1595$.

Cohn, T. E., Green, D. G. \& Tanner, Jr, W. P. (1975). Receiver operating characteristic analysis. Application to the study of quantum fluctuation effects in optic nerve of Rana pipiens. Journal of General Physiology, 66, 583-616.

Copenhagen, D. R., Donner, K. \& Reuter, T. (1987). Ganglion cell performance at absolute threshold in toad retina: effects of dark events in rods. Journal of Physiology, 393, 667-680.

Curcio, C. A., Sloan, K. R., Kalina, R. E. \& Hendrickson, A. E. (1990). Human photoreceptor topography. Journal of Comparative Neurology, 292, 497-523.

Dartnall, H. J. A. (1972). Photosensitivity. In Dartnall, H. J. A. (Ed.), Handbook of sensory physiology (Vol. VII/I, pp. 122-145). Berlin: Springer.

Dartnall, H. J. A., Bowmaker, J. K. \& Mollon, J. D. (1983). Human visual pigments: Microspectrophotometric results from the eyes of seven persons. Proceedings of the Royal Society B, 220, 115-130.

Davila, K. D. \& Geisler, W. S. (1991). The relative contributions of pre-neural and neural factors to areal summation in the fovea. Vision Research, 31, 1369-1380.
Donner, K. (1989). The absolute sensitivity of vision: Can a frog become a perfect detector of light-induced and dark rod events? Physica Scripta, 39, 133-140.

Donner, K., Firsov, M. L. \& Govardovskii, V. I. (1990). The frequency of isomerisation-like "dark" events in rhodopsin and porphyropsin rods of the bullfrog retina. Journal of Physiology, 428, 673-692.

Fechner, G. T. (1860). Elemente der Psychophysik. Leipzig: Breitkopf und Härtel.

Firsov, M. L. \& Govardovskii, V. I. (1990). Dark noise of visual pigments with different absorption maxima. Sensory Systems, 4, 25-34. (In Russian.)

Hallett, P. E. (1962). Scotopic acuity and absolute threshold in brief flashes. Journal of Physiology, 163, 175-189.

Hallett, P. E. (1963). Spatial summation. Vision Research, 3, 9-24.

Hallett, P. E. (1969a). Impulse functions for human rod vision. Journal of Physiology, 202, 379-402.

Hallett, P. E. (1969b). The variations in visual threshold measurement. Journal of Physiology, 202, 403-419.

Hallett, P. E. (1969c). Quantum efficiency and false positive rate. Journal of Physiology, 202, 421-436.

Hallett, P. E. (1987). Quantum efficiency of dark-adapted human vision. Journal of the Optical Society of America, A, 4, 2330-2335.

Hallett, P. E., Marriott, F. H. C. \& Rodger, F. C. (1962). The relationship of visual threshold to retinal position and area. Journal of Physiology, 160, 364-373.

Hecht, S., Shlaer, S. \& Pirenne, M. H. (1942). Energy, quanta and vision. Journal of General Physiology, 25, 819-840.

Herrick, R. M. (1956). Foveal luminance discrimination as a function of the duration of the decrement or increment in luminance. Journal of Comparative Physiology and Psychology, 49, 437-443.

Hillmann, B. M. (1958). Relationship between stimulus size and threshold intensity in the fovea measured at four exposure times. Journal of the Optical Society of America, 48, 422-428.

Hirsch, J. \& Curcio, C. A. (1989). The spatial resolution capacity of human foveal retina. Vision Research, 29, 1095-1101.

Kolb, H. (1991). Anatomical pathways for color vision in the human retina. Visual Neuroscience, 7, 61-74.

Lamb, T. D. \& Simon, E. J. (1977). Analysis of electrical noise in turtle cones. Journal of Physiology, 272, 435-468.

MacLeod, D. I. A., Williams, D. R. \& Makous, W. (1985). Difference frequency gratings above the resolution limit. Investigative Ophthalmology and Visual Science, 26 (Suppl.), 11.

Makous, W. (1990). Absolute sensitivity. In Hess, R. F., Sharpe, L. T. \& Nordby, K. (Eds), Night vision (pp. 146-176). Cambridge: Cambridge University Press.

Marriott, F. H. C. (1963). The foveal absolute visual threshold for short flashes and small fields. Journal of Physiology, 169, 416-423.

Polyak, S. L. (1941). The retina. Chicago: The University of Chicago Press.

Østerberg, G. (1935). Topography of the layer of rods and cones in the human retina. Acta Ophthalmologica, 13, 1-103.

Sakitt, B. (1971). Configuration dependence of scotopic spatial summation. Journal of Physiology, 216, 513-529.

Sakitt, B. (1972). Counting every quantum. Journal of Physiology, 223, 131-150.

Schnapf, J. L., Nunn, B. J., Meister, M. \& Baylor, D. A. (1990). Visual transduction in cones of the monkey Macaca fascicularis. Journal of Physiology, 427, 681-713.

Scholtes, A. M. W. \& Bouman, M. A. (1977). Psychophysical experiments on spatial summation at threshold level of the human peripheral retina. Vision Research, 17, 867-873.

Sharpe, L. T., Fach, C. \& Nordby, K. N. (1988). Temporal summation in the achromat. Vision Research, 28, 1263-1269.

Sharpe, L. T., Fach, C., Nordby, K. \& Stockman, A. (1989). The incremental threshold of the rod system and Weber's law. Science, 244, 354-356.

Snyder, A. W. \& Pask, C. (1973). The Stiles-Crawford effectexplanation and consequences. Vision Research, 13, 1115-1137.

Stiles, W. S. (1948). The physical interpretation of the spectral sensitivity curve of the eye. In Transactions of the optical convention 
of the worshipful company of spectacle makers (pp. 97-107). London: Spectacle Makers' Co.

Stiles, W. S. (1949). Investigations of the scotopic and trichromatic mechanisms of vision by the two-colour threshold technique. Revue d'Optique, 28, 215-237.

Stiles, W. S. (1953). Further studies of visual mechanisms by the two-colour threshold method. In Union international de physique pure et appliquée: Coloquio sobre problemas opticos de la vision (pp. 65-103). Madrid: Instituto de Optica "Daza de Valdes".

van der Velden, H. (1944). Over het antaal lichtquanta dat nodig es for en licht prickel bij dat menselyk oog. Physica, 11, 179-189.

Vimal, R. L. M., Pokorny, J., Smith, V. C. \& Shevell, S. K. (1989). Foveal cone thresholds. Vision Research, 29, 61-78.

Vos, J. J., Walraven, J. \& van Meeteren, A. (1976). Light profiles of the foveal image of a point source. Vision Research, 16, 215-219.

Walraven, P. L. (1966). Recovery from the increase of the Stiles-Crawford effect after bleaching. Nature, 210, 311-312.

Walraven, P. L. \& Bouman, M. A. (1960). Relation between directional sensitivity and spectral response curves in human vision. Journal of the Optical Society of America, 50, 780-784.
Wässle, H., Grünert, U., Röhrenbeck, J. \& Boycott, B. (1989). Cortical magnification factor and the ganglion cell density of the primate retina. Nature, 341, 643-646.

Weinstein, C. \& Arnulf, A. (1946). Contribution à l'étude des seuils de perception de l'oeil. Communications de l'Institut d'Optique de Paris, 2, 1-43.

Wesner, M. F., Pokorny, J., Shevell, S. K. \& Smith, V. C. (1991). Foveal cone detection statistics in color-normals and dichromats. Vision Research, 31, 1021-1037.

Williams, D. R., MacLeod, D. I. A. \& Hayhoe, M. M. (1981a). Foveal tritanopia. Vision Research, 21, 1341-1356.

Williams, D. R., MacLeod, D. I. A. \& Hayhoe, M. M. (1981b). Punctate sensitivity of the blue-sensitive mechanism. Vision Research, 21, 1357-1375.

Zacks, J. L. (1970). Temporal summation phenomena at threshold: Their relation to visual mechanisms. Science, 170, 197-199.

Acknowledgements - I wish to thank Professor Tom Reuter for critically reading the manuscript. This work was supported by the Academy of Finland (grants $01 / 455$ and $01 / 1011872$ ). 